\title{
Measuring the LISA test mass magnetic proprieties with a torsion pendulum
}

\author{
M Hueller ${ }^{a}$, M Armano ${ }^{a, b}$, L Carbone $^{a}$, A Cavalleri $^{c}$, \\ R Dolesi ${ }^{a}$, C D Hoyle ${ }^{a} \dagger$, S Vitale ${ }^{a}$ and $\mathbf{W} \mathbf{J}$ Weber $^{a}$ \\ ${ }^{a}$ Dipartimento di Fisica, Università di Trento and INFN, Sezione di Padova, \\ Gruppo Collegato di Trento, I-38050, Povo, Trento, Italy \\ ${ }^{b}$ Dipartimento di Fisica, Università dell'Insubria, I-22100, Valleggio, Como, \\ Italy \\ ${ }^{c}$ CEFSA-ITC, I-38050, Povo, Trento, Italy \\ E-mail: hueller@science.unitn.it
}

\begin{abstract}
Achieving the low frequency LISA sensitivity requires that the test masses acting as the interferometer end mirrors are free-falling with an unprecedented small degree of deviation. Magnetic disturbances, originating in the interaction of the test mass with the environmental magnetic field, can significantly deteriorate the LISA performance and can be parameterized through the test mass remnant dipole moment $\vec{m}_{r}$ and the magnetic susceptibility $\chi$. While the LISA test flight precursor LTP will investigate these effects during the preliminary phases of the mission, the very stringent requirements on the test mass magnetic cleanliness make ground-based characterization of its magnetic proprieties paramount. We propose a torsion pendulum technique to accurately measure on ground the magnetic proprieties of the LISA/LTP test masses.
\end{abstract}

PACS numbers: 04.80.Nn, 07.55.Jg, 07.87.+v

\section{Introduction}

The LISA (Laser Interferometer Space Antenna) sensitivity goal requires that the test masses (nominally $2 \mathrm{~kg}$ ) are kept in free fall with an acceleration noise below $3 \mathrm{fm} \mathrm{s}^{-2} \mathrm{~Hz}^{-1 / 2}$ at frequencies down to $0.1 \mathrm{mHz}$ 1]. In order to achieve this high purity of geodesic motion, environmental noisy forces are screened by shielding the test masses in a drag-free satellite, with precision thrusters driven by a position sensor in order to minimize the test mass - satellite relative displacement. Among the residual disturbance sources, magnetic effects play a paramount role, as discussed in [2, 3]: the fluctuations of both magnetic field and magnetic field gradient will couple to the test mass remnant dipole moment $\vec{m}_{r}$ and susceptibility $\chi$, to produce force noise. In the limit of weakly magnetic materials, the component of the force acting on the mass along the LISA interferometer axis $x$ can be expressed as

$$
f_{x}(t) \approx\left(\vec{m}_{r}+\frac{\chi V}{\mu_{0}} \vec{B}(t)\right) \cdot \frac{\partial}{\partial x} \vec{B}(t)=\vec{m}_{r} \cdot \frac{\partial}{\partial x} \vec{B}(t)+\frac{\chi V}{\mu_{0}} \vec{B}(t) \cdot \frac{\partial}{\partial x} \vec{B}(t)(1)
$$

with similar relations holding for $f_{y}(t)$ and $f_{z}(t)$. Here we describe the test mass magnetic proprieties by its permanent, remnant magnetic dipole moment $\vec{m}_{r}$ and

$\dagger$ Currently at the Department of Physics, University of Washington, Seattle, WA 98195 USA 
its magnetization induced through the (small) magnetic susceptibility $\chi$ by the externally applied magnetic field $\vec{B}(t)$. For LISA, fluctuations of $\vec{B}(t)$ are expected to be dominated by the interplanetary magnetic field, while fluctuations of the magnetic field gradient $\partial \vec{B}(t) / \partial x$ are expected to be produced by sources on the satellite itself. In order to relax the consequent environmental requirements on the satellite, it is crucial to obtain a test mass with very good magnetic proprieties; for LISA the requirements are $\left|\vec{m}_{r}\right| \leqslant 10 \mathrm{nA} \mathrm{m}$ and $^{2}|\chi| \leqslant 3 \cdot 10^{-6}$. The test mass design calls for a $70 \% \mathrm{Au}-30 \% \mathrm{Pt}$ alloy, with composition chosen in order to achieve the lowest susceptibility, while retaining high density to minimize the displacement caused by a given force disturbance. The characterization of the full-sized test mass for LISA and its flight precursor LTP [5], particularly important given the very stringent requirements on the magnetic cleanliness level, is made difficult with the standard magnetic characterization techniques, such as SQUID magnetometers and susceptometers, by its relatively large dimensions.

In this article we discuss an application of a high sensitivity torsion pendulum facility, developed for several testing of force disturbances for LISA [6] 7], 8], to the independent characterization of both the test mass remnant moment and susceptibility, assessing these proprieties directly through the forces and torques associated with the variation of magnetic fields.

\section{Characterizing a LISA test mass with a torsion pendulum}

In the proposed experiment we will measure with high resolution the AuPt test mass remnant moment $\vec{m}_{r}$ and susceptibility $\chi$, by exploiting the high torque sensitivity of a torsion pendulum where the test mass is included in a light, non magnetic holder and is suspended by a thin fiber, as sketched in figure 1 The magnetic proprieties of the LISA test mass will be measured by observing the torques acting on it when subjected to a controlled oscillating field $\vec{B}_{\mathrm{f}}(\vec{x}, t)=\vec{B}_{0}(\vec{x}) \sin \omega_{m} t$ produced by a suitable excitation coils configuration. Assuming the addition of the external, residual dc magnetic field $\vec{B}_{\text {res }}$, the total applied field is then $\vec{B}(\vec{x}, t)=\vec{B}_{\text {res }}(\vec{x})+\vec{B}_{0}(\vec{x}) \sin \omega_{m} t$. In order to evaluate the effect of the applied magnetic field we need to account 3 components of $\vec{B}_{0}$ and $\overrightarrow{B_{\text {res }}}$, which depend on position within the test mass $\vec{x}=\left(x_{i}, y_{i}, z_{i}\right)$. The test mass is then modelled by meshing it into a grid of small elements with volume $\mathcal{V}$, located at the positions $\vec{x}$, each small enough to assume locally uniform field $\vec{B}(t)=\left(B_{x}(t, \vec{x}), B_{y}(t, \vec{x}), B_{z}(t, \vec{x})\right)$. Each element $i$ interacting with the externally applied magnetic field is characterized by a remnant magnetic moment $d \vec{m}_{r}(\vec{x})$ (with $\left.\sum_{i} d \vec{m}_{r}(\vec{x})=\vec{m}_{r}\right)$ and a susceptibility $\chi(\vec{x})$. The force along the $x$ axis acting on each test mass element can then be expressed using (11) as a combination of a dc term

$$
f_{x \mathrm{dc}} \approx\left(d \vec{m}_{r}+\frac{\chi \mathcal{V}}{\mu_{0}} \vec{B}_{\mathrm{res}}\right) \cdot \frac{\partial}{\partial x} \vec{B}_{\mathrm{res}}+\frac{\chi \mathcal{V}}{2 \mu_{0}} \vec{B}_{0} \cdot \frac{\partial}{\partial x} \vec{B}_{0},
$$

a term at the modulation frequency $f_{m}=\omega_{m} / 2 \pi$

$$
f_{x 1 \omega_{m}} \approx\left[\left(d \vec{m}_{r}+\frac{\chi \mathcal{V}}{\mu_{0}} \vec{B}_{\text {res }}\right) \cdot \frac{\partial}{\partial x} \vec{B}_{0}+\frac{\chi \mathcal{V}}{\mu_{0}} \vec{B}_{0} \cdot \frac{\partial}{\partial x} \vec{B}_{\text {res }}\right] \sin \omega_{m} t
$$

and a term at twice the modulation frequency $2 f_{m}$

$$
f_{x 2 \omega_{m}} \approx-\frac{\chi \mathcal{V}}{2 \mu_{0}}\left(\vec{B}_{0} \cdot \frac{\partial}{\partial x} \vec{B}_{0}\right) \cos 2 \omega_{m} t .
$$


The torque around the vertical $z$ axis running through the test mass center of mass is given instead by the interaction of the horizontal $(x, y)$ projections of the remnant magnetic moment, $d \vec{m}_{r}$, with the total applied magnetic field:

$$
\overrightarrow{n_{z}}(t) \approx\left(d \vec{m}_{r} \times \vec{B}(t)\right)_{z},
$$

and can be written as a combination of a dc term

$$
{\overrightarrow{n_{z}}}_{\mathrm{dc}}=\left(d \vec{m}_{r} \times \vec{B}_{\mathrm{res}}\right)_{z}
$$

and a $1 f_{m}$ term

$$
\vec{n}_{z 1 \omega_{m}}(t)=\left(d \vec{m}_{r} \times \vec{B}_{0}\right)_{z} \sin \omega_{m} t
$$

while the $2 f_{m}$ component vanishes because the magnetic moment induced through the susceptibility $\chi$ is parallel to the applied field. After evaluating the forces acting on each test mass element $f_{x}\left(x_{i}, y_{i}, z_{i}\right)$ and $f_{y}\left(x_{i}, y_{i}, z_{i}\right)$ given by (11), together with the torque about the $z$ axis $n_{z}\left(x_{i}, y_{i}, z_{i}\right)$ given by (5), we account also for the torque arising from the net force difference at different locations within the test mass due to the remnant and induced magnetic moment:

$$
\tilde{N}_{i z}(t) \approx x_{i} f_{y}\left(t, x_{i}, y_{i}, z_{i}\right)-y_{i} f_{x}\left(t, x_{i}, y_{i}, z_{i}\right) .
$$

Here $x_{i}$ and $y_{i}$ are the horizontal distances of the element from the torsional axis.

For given external and applied fields, the overall torque about the $z$ axis $N_{z}$, which the torsion pendulum is sensitive to, is then evaluated by adding the contributions from all the mass elements $i$ :

$$
N_{z}(t)=\iiint_{V}\left(n_{i z}(t)+\tilde{N}_{i z}(t)\right) \mathrm{d} x \mathrm{~d} y \mathrm{~d} z=\sum_{i}\left(n_{i z}(t)+\tilde{N}_{i z}(t)\right) .
$$

Again, the torque can be read as the superposition of terms at dc, $1 f_{m}$ and $2 f_{m}$.

\subsection{Remnant moment $\vec{m}_{r}$}

The test mass remnant magnetic moment $\vec{m}_{r}$ can be measured from the torque it feels by an uniform oscillating magnetic field $\vec{B}_{0} \sin \omega_{m} t$. The torque in (9) becomes then

$$
\begin{aligned}
& N_{z}(t)=\sum_{i} n_{i z}(t)=\left(\sum_{i} d \vec{m}_{r}\left(x_{i}, y_{i}, z_{i}\right)\right) \times\left(\vec{B}_{\mathrm{res}}+\vec{B}_{0} \sin \omega_{m} t\right)= \\
& =\vec{m}_{r} \times\left(\vec{B}_{\mathrm{res}}+\vec{B}_{0} \sin \omega_{m} t\right)
\end{aligned}
$$

and can be detected by coherent demodulation of the fiber angular deflection $\phi(t)$ at the excitation frequency $f_{m}=\omega_{m} / 2 \pi$, through the torsion pendulum transfer function

$$
\phi(\omega)=N(\omega) F(\omega)=N(\omega)\left[\Gamma\left(1-\left(\omega / \omega_{0}\right)^{2}+i / Q\right)\right]^{-1} .
$$

Here $\omega_{0}=\sqrt{\Gamma / I}$ is the pendulum resonance frequency, $\Gamma=\Gamma_{\mathrm{f}} \cdot(1+i \delta)$ is the torsional spring constant, $Q=1 / \delta$ is the pendulum mechanical quality factor, given by the inverse of the fiber loss angle $\delta$. This experiment is routinely performed as a preliminary step to evaluate the impact of the laboratory magnetic noise on high sensitivity torsion pendula performance [3, 6].

The expected torque sensitivity of the proposed torsion pendulum, assuming a thermal torque noise spectrum for a quality factor $Q=1000$, is shown in figure 1 The maximum sensitivity is set by the combination of the optical readout and the pendulum thermal noise at $S_{N}^{1 / 2} \approx 30 \mathrm{fN} \mathrm{m} \mathrm{Hz}{ }^{-1 / 2}$ around $10 \mathrm{mHz}$. Applying an oscillating 


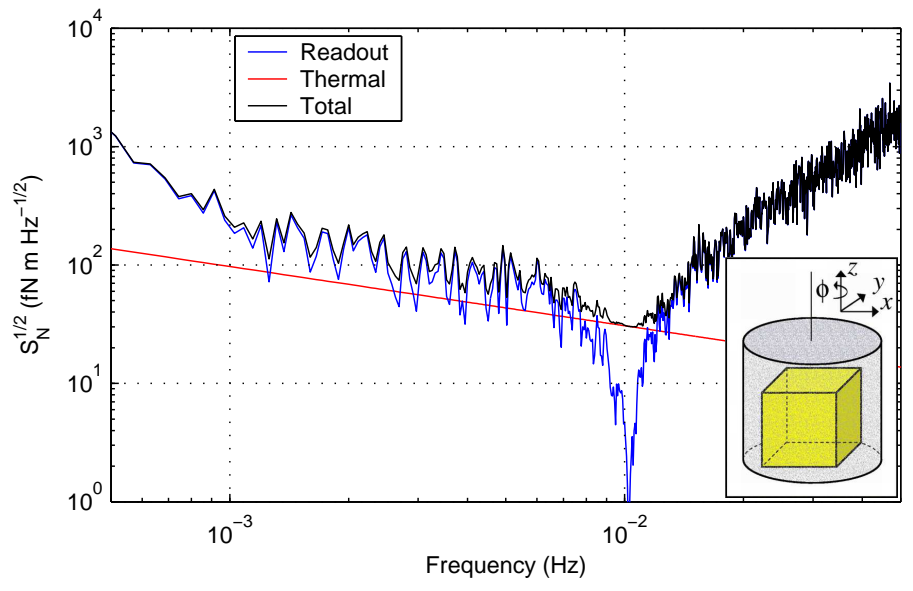

Figure 1. Expected torque noise floor for a torsion pendulum, comprised of a commercial W fiber with length $L=1 \mathrm{~m}$, diameter $d=125 \mu \mathrm{m}$, supporting along its axis one LISA cubic test mass, with side $L=46 \mathrm{~mm}$, mass $M=1.95 \mathrm{~kg}$, enclosed in a cylindrical $\mathrm{Al}$ holder as sketched in the inset. The pendulum thermal noise, conservatively assuming a quality factor $Q=1000$, is combined with the angular noise of the commercial autocollimator currently installed on the torsion pendulum facility 3 6, converted into equivalent mechanical noise through the torsion pendulum transfer function 11. This noise performance can be applied to the measurement of both the test mass remnant moment $\vec{m}_{r}$ and susceptibility $\chi$, described in section 2.1 and 2.2 respectively.

magnetic field with amplitude $\left|\vec{B}_{0}\right|=10 \mu \mathrm{T} \S$, and assuming 3 hour integration time, this noise performance yields an expected remnant moment resolution of $\left|\Delta \vec{m}_{r}\right| \approx$ $40 \mathrm{pA} \mathrm{m}^{2}$, well below the LISA requirements. Suitable flipping of the test mass within the holder will allow measurement of all components of the magnetic moment $\vec{m}_{r}$. To take advantage of this resolution, the remnant moment of the sample holder, without test mass, should be measured and subtracted (typical values for torsion pendula are $\left.\left|\vec{m}_{r}\right| \approx 1-10 \mathrm{nA} \mathrm{m}^{2}[3,9]\right)$.

\subsection{Susceptibility $\chi$}

Assuming homogeneous test mass, the $2 f_{m}$ torque component in (9) can be written as

$$
N_{z 2 \omega_{m}}=-\frac{\chi \mathcal{V}}{2 \mu_{0}} \sum_{i} \vec{B}_{0}(\vec{x}) \cdot\left(x_{i} \frac{\partial}{\partial y} \vec{B}_{0}(\vec{x})-y_{i} \frac{\partial}{\partial x} \vec{B}_{0}(\vec{x})\right) \cos 2 \omega t .
$$

Applying a forcing magnetic field pattern which exerts different forces on opposing test mass sides, it is then possible to single out the effect induced by the susceptibility $\chi$ as a net torque signal $N_{z 2 \omega_{m}}$ at twice the excitation frequency $f_{m}$ directly proportional to the test mass average susceptibility $\chi$. Any effect from the remnant moment will not directly couple to this measurement and will appear only in $N_{z 1 \omega_{m}}$. A realistic configuration, compatible with the dimensions of the facility vacuum vessel,

$\S$ This excitation field configuration can be for instance produced by means of a set of Helmholtz coils with radius $R=45 \mathrm{~cm}$, wound with $n=40$ turns and powered with currents $I=100 \mathrm{~mA}$. The coil size is sufficient to ensure that the field gradient over the extension of the test mass is negligible. 


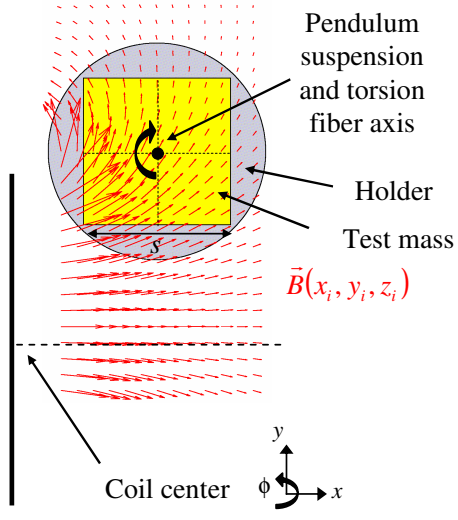

(a)

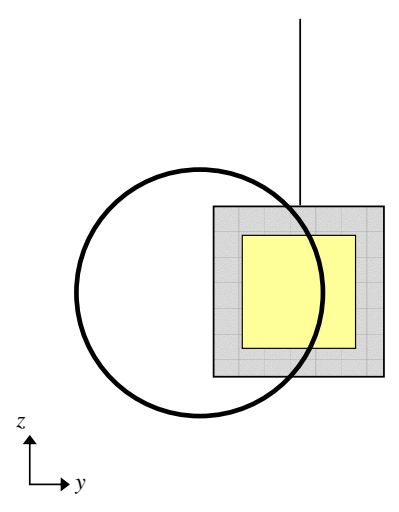

(b)

Figure 2. Source coil arrangement for test mass susceptibility $\chi$ measurement. (a) Top view, with the test mass displaced with respect to the coil axis and to the coil plane. The red arrows represent the components of the applied magnetic field in the horizontal plane $B_{0 x}$ and $B_{0 y}$. (b) Side view. The single coil configuration creates a magnetic field and field gradient which differ on opposite sides of the test mass, inducing then a net $2 f_{m}$ torque $N_{z 2 f_{m}}(t) \propto \chi$. The relative test mass - coil position has been chosen in order to maximize the susceptibility induced torque (see figure 3 .

is described in figure 2 and employs a relatively small coil, with symmetry axis placed away from the pendulum torsion axis. In the case of a test mass average susceptibility $\chi=3 \cdot 10^{-6}$, the expected induced torque at twice the excitation frequency is shown in figure 3] and has a maximum of $\left|N_{z 2 \omega_{m}}\right| \approx 26.5 \mathrm{fN} \mathrm{m}$. Assuming the same torque noise level and a 3 hour measurement as in section 2.1 this signal amplitude will permit a resolution $|\Delta \chi| \approx 5 \cdot 10^{-8}$, corresponding to $\lesssim 2 \%$ of the LISA goal.

The measurement resolution grows as $\vec{B}_{0} \cdot \frac{\partial}{\partial x} \vec{B}_{0} \propto I^{2}$, so it is possible to effectively improve the signal to noise ratio by increasing the source coil drive current $I$. However, the major uncertainty is given here by the model assumed to evaluate the magnetic field and field gradients, and thus to estimate the forces and torques. Nevertheless, the power of the technique is evident because it will allow characterization of the LISA test mass directly from the torques exerted by time-depending magnetic fields,

and independent estimate of the material proprieties to be compared with other characterization methods.

\section{Conclusions}

The wide range of properties requested for the LISA test masses (good optical quality to serve as end mirrors of the interferometers, stringent machining tolerances to avoid stray cross coupling, high mechanical strength to sustain the launch vibrations, high density and homogeneous composition to minimize acceleration for given force, good magnetic cleanliness) makes its production a fundamental process within the LISA technology development. In addition, the strict requirements on the magnetic cleanliness make the verification of these proprieties a very important issue. Even if a comprehensive testing campaign is planned during the preliminary phases of the flight, 


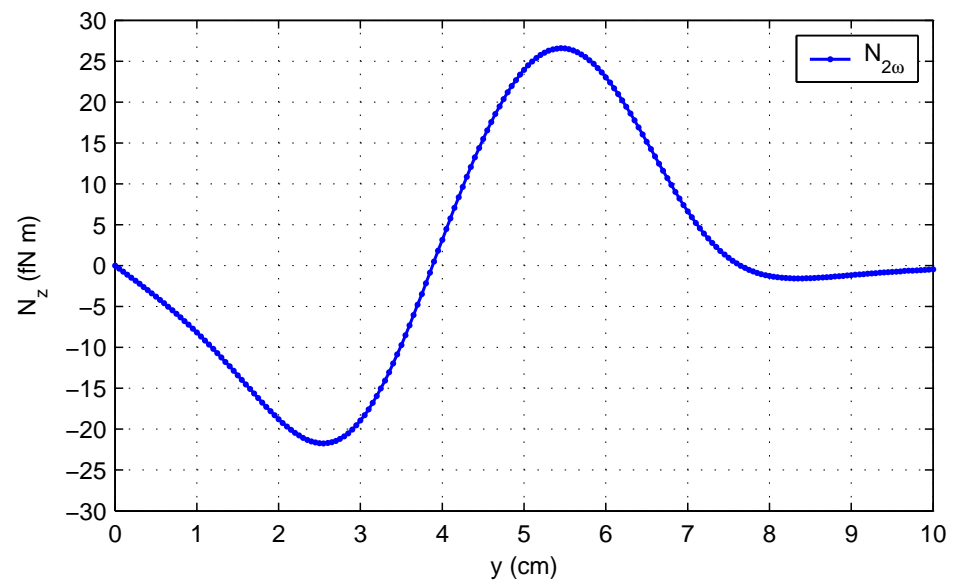

Figure 3. The susceptibility-induced signal $N_{z 2 f_{m}}(t)$ in (12), due to the interaction of the test mass with the magnetic field produced by the coil in the arrangement sketched in figure 2 as a function of the displacement $y$ between the test mass and the coil axis. The coil parameters are: radius $R=5 \mathrm{~cm}$, $n=4$ turns, excitation current $I=2 \mathrm{~A}$, on-axis displacement $x=4 \mathrm{~cm}$; the maximum torque is obtained with off-axis displacement $y=5.5 \mathrm{~cm}$.

with the aim of establishing the "feedthrough" of the magnetic field fluctuations to acceleration noise [5, a ground based experimental investigation of the force/torques associated with varying magnetic field is highly desirable. The torsion pendulum technique, with its widely demonstrated high torque sensitivity, can be applied to a significant characterization of the magnetic proprieties of the LISA/LTP test masses. The principle of operation, based on the coherent detection of small torques associated with modulation of external magnetic fields, is analogous to the test procedure to be employed during the flight, and thus represents an important validation step in view of the mission. Work is currently in progress to modify the existing facility in order to host the proposed experiment, and modelling is being performed in order to assess the validity of the analysis and the method performance.

\section{Acknowledgments}

It is a pleasure to acknowledge many fruitful discussions with E Adelberger, J Mester, D L Gill, S Anza, A Sanchez, D Chen.

\section{References}

[1] P Bender et al 2000 LISA ESA-SCI(2000)11 System and Technology Study Report

[2] S Vitale et al 2002 LISA Technology Package Architect Final Report (ESTEC 15580/01/NL/HB)

[3] M Hueller 2003 Phd Thesis University of Trento

[4] J Hanson et al 2003 Class. Quantum Grav. 20 S109

[5] D Bortoluzzi et al 2004 Class. Quantum Grav. 21 S573

[6] L Carbone et al 2004 Class. Quantum Grav. 21 S611

[7] L Carbone et al 2003 Phys. Rev. Lett. 91151101

[8] L Carbone et al submitted to Class. Quantum Grav.

[9] C D Hoyle et al 2004 Phys. Rev. D $\mathbf{7 0} 042004$ 